\title{
Semantic Keys and Reading *
}

\author{
Zev bar-Lev ${ }^{* *}$ \\ (San Diego State University, USA)
}

\begin{abstract}
Semantic Keys are elements (word-parts) of written language that give an iconic, general representation of the whole word's meaning. In written Sino-Japanese the "radical" or semantic components play this role. For example, the character meaning "woman, female' is the Semantic Key of the character for Ma 'Mama' (alongside the phonetic component $M a$, which means 'horse' as a separate character). The theory of semantic Keys in both graphic and phonemic aspects is called qTheory or nanosemantics.

The most innovative aspect of the present article is the hypothesis that, in languages using alphabetic writing systems, the role of Semantic Key is played by consonants, more specifically the first consonant. Thus, $L$ meaning 'LIFT' is the Semantic Key of English Lift, Ladle, Lofty, aLps, eLevator, oLympus; Spanish Leva, Lecantarse, aLto, Lengua; Arabic aLLah, and Hebrew ${ }^{(1)}$ a $e L^{-} a L$ 'upto-above' (the Israeli airline), Polish Lot 'flight' (the Polish airline); Hebrew 'eL, 'eLohim 'God', and haLLeluyah 'praise-ye God' (using Parallels, 'Lift up God'). Evidence for the universality of the theory is shown by many examples drawn from various languages, including Indo-European Semitic, Chinese and Japanese. The theory reveals hundreds of relationships within and between languages, related and unrelated, that have been "Hiding in Plain Sight", to mention just one example: the Parallel between Spanish Pan 'bread' and Mandarin Fan 'rice'.
\end{abstract}

\footnotetext{
* In this article and in all things, I am grateful for the support and participation of my wife and associate researcher, Shoshana. I am also grateful for suggestions on a previous version of this article by my some-time co-author Arthur Palacas.

${ }^{* *}$ Zev bar-Lev: Professor of Hebrew and Linguistics, Department of Linguistics, the College of Arts \& Letters, San Diego State University, 7996 Laurelridge Road, San Diego, CA 92120, USA. E-mail: Zev.bar-Lev@SDSU.edu. Zev bar-Lev is the creator of "Sheltered Initiation Language Learning" method. He has published research in several fields of theoretical linguistics (semantics, pragmatics, functional syntax, discourse theory, morpho-phonology, linguistics and computers) on (and in) several languages (Hebrew, Russian, and others), and the researches were published in Glossa (1968, 1972, 1975), Philo (1975), Linguistics (1977, 1978), Folia Linguistica (1977, 1978, 1984), Lingua (1978, 1980), Afroasiatic Linguistics (1978), International Review of Slavic Linguistics (1979), Visible Language (1983), International Journal Of The Humanities (2003), Noetic Journal (2003), Journal of Arabic \& Islamic Studies (2005), etc.

(1) Note that Hebrew - a here are silent consonants (abstract phonemes which influence surrounding vowels).
} 
Keywords: Lexicon, Semantic Theory, Key-letters, ambiguity

\section{Preview}

This intentionally short article briefly but comprehensively outlines qTheory, or nanosemantics. This is a radical, even "heretical" theory of lexical semantics, positing SEMANTIC KEYS with kinesthetic-iconic value, which explain hundreds of relationships that have been "hiding in plain sight", within and between various genetically unrelated languages, to mention just one example: the Parallel between Spanish Pan 'bread' and Mandarin Fan 'rice' or even more startlingly the Hebrew plural cherub-iM and the Chinese plural-haidz-Men.

To "prove" their universality definitively would require long study of dozens of individual lexicons, so we here made a prima-facie case for positing the theory, in hopes of providing other researchers with new tools for lexical-semantic analysis. This prima-facie case will use various unrelated non-Indo-European languages, as well as English (both Germanic and Latinate Lexicon).

\section{1 qTheory \& qLexicon}

"Nanosemantics" (or "qTheory", Where $q$ means "semantic Key") is here initially proposed (1) as a theory of alphabetic writing and of the cognitive (especially lexical) structures behind reading process. More precisely it can be called a theory of the implicit (subconscious) Lexicon of written language (the wLexicon or "qLexicon"). After explaining the theory vis-à-vis written language, links will be set forth to construe (2) an implied theory of language generally, in fact as a universal lexical-semantic theory. The case for its universality is made by its initial formulation for one non-Indo-European language, and its final applicability to other non-IE languages, its applicability to IE languages being an implicit intermediate step.

The meaning-centered view behind nanosemantics especially typified an earlier article on the meaning of the conjunction and (bar-Lev \& Palacas, 1980). The present article supersedes my earlier analysis of Arabic (bar-Lev, 2005-6).

\section{2 qLexicon $=$ wLexicon $=$ sLexicon}

This formula represents the unification to be posited of the various linguistic levels initially distinguished here:

A fundamental distinction underlying this article is the distinction between spoken language ("sLanguage", Mandarin $h w a$ ). And written language ("wLanguage", Mandarin wen). We will be proposing that qLexicon, the lexicon of theory of wLanguage, is in fact also the sLexicon, the lexicon of spoken language.

In fact we will be modifying de Saussure's axioms defining linguistics. That is, by formulating a theory of written language, we may be going beyond narrowly defined, but 
qTheory does belong to linguistics in the larger sense of German Sprachwissenschaft or Russian yazikovedenie. But it would be a mistake to dismiss it as "non-linguistic" or "just semiotics", since lexicon belongs to core linguistics: one cannot be linguistically competent on the basis of syntax and phonology alone!

As noted, the theory can conveniently be called qTheory (where "q" stands for a Semantic Key, or it can be called "nanosemantics" on the analogy of nanosyntax (Starke, 2009), from which it derives the assumption (itself heretical) of sub-morphemes, i.e. that lexical items are often syntactically complex. More specifically, it will be proposed here that lexical items generally consist of a Semantic Key plus a "rhyme" — generally meaningless. The Key and Rhyme parallel to the semantic vs. phonetic portion of Sino-Japanese characters.

The basic idea can be expressed simply. The central claim will be that the first consonant of any word in any alphabetic written language is an iconic Semantic key for the meaning of the whole word, much as the semantic portion or "radical" of many Sino-Japanese compound characters gives a clue to the meaning of the compound character.

\subsection{Semantic Keys \& Chinese "radicals"}

The main hypothesis defining nanosemantics is that of semantic Keys, a linguistic unit hypothesized here, which serves as a "determinative" to words of written language. "Hiding in plain view" like linguistic structure generally, these Keys give a "helpful hint" about the meaning of each printed word, just as the "radical" (semantic part) of Sino-Japanese characters does.

For example, the radical MOUTH, a squared circle, is used in characters meaning 'say, eat', while the HEART radical is used in characters meaning 'think, love'. Similarly WOMAN (picturing a seated woman) combines with the phonetic Ma 'horse' to give Mama 'Mom' (not 'female horse'). The phonetic part of the Sino-Japanese character is paralleled by the remainder of the word, the "rhyme". So English Hunker and Bunker have Keys $\sqrt{H}_{\mathrm{H}}$ and $\sqrt{\mathrm{B}}$ (meaning HUG \& BULGE respectively), while sharing the rhyme -unker, which is meaningless.

The semantic Keys will be hypothesized here as the organizing basis of the qLexicon. explaining theoretically the way reading works (for the "ideal reader") and therefore having various important practical applications, in overcoming obstacles to literacy, whether for special-needs or ordinary children, or adults with reading difficulties of various extent and cause, and the special audience of the deaf, for whom mainstream remedies like teaching phonological awareness may well be counterproductive (as many specialists already know). Learning to read in foreign languages (FLs) is also an area of especially worthy applications, using the "Global Alphabet" defined by the theory. We will later propose that they also belong to "narrow" linguistics, in that they derive from an oral precursor of Key 
consonant phonemes.

The Keys as phonemes might seem to be onomatopoetic. Certainly $S h$ seems onomatopoetic in Hebrew Sheqet 'silence', Shalom 'peace', and Russian Shopot 'whisper'. But more generally the Keys are kinesthetic: $L$ does not sound like Lifting, but the tongue (Lingua) does Lift in pronouncing L. That qTheory applies not only to wLanguage is assured by the almost complete parallels (as shown in the equation above) between the spoken-language and written-language lexicons (the sLexicon or qLexicon and the wLexicon).

\section{Origin of qTheory}

The Core of the theory was initially developed for just one Non-Indo-European language, Hebrew. It was later found to apply broadly, not only to Arabic (another Semitic language) but also to English, including both Latinate and Germanic roots. Later it was seen to apply, so far partially but with surprising specificity, to Chinese (Mandarin and Cantonese) and also Japanese.

For Hebrew, several decades of research yielded a theory of "Key-letters", described in various linguistic publications in Israel and the US, written in Hebrew and English, covering Hebrew (and Arabic).

This theory is presented in tabular form in table 1. Each Hebrew letter (given in transliteration) is given two "Main Meanings", $\mathrm{mm} 1$ and $\mathrm{mm} 2$. Both meanings are posited as the basis for reading and aural comprehension, via the process of "tweaking:" (and "super-tweaking"); furthermore $\mathrm{mm} 2$ is tweaked from $\mathrm{mm} 1$.

Tweaking is the process whereby Hebrew kaf is understood to mean 'spoon' or 'palm of the hand' or 'shovel', depending on context.

"Super-tweaking" is the process by which any Hebrew word whose first consonant is the letter can mean a CONTAINER (a glass tumbler, pocket, chair, church, congress/convention) or CONTAINING PLACE, or a verb of CONTAINMENT, INCLUSION, or CONTAINING, or adjectives of ABILITY ('able').

Table 1. Short Table of Hebrew Keys

\begin{tabular}{|l|l|l|l|}
\hline Hebrew Keys (transliterated) & Roman/IE equiv. & Mm1 & Mm2 \\
\hline $\mathbf{M}, \mathbf{W}$ & $\mathbf{M}, \mathbf{W}$ & PULL & TOGETHER \\
\hline $\mathbf{H}, \mathbf{C K}, \mathbf{Q}$ & $\mathbf{H}, \mathbf{C} / \mathbf{K}, \mathbf{Q}$ & CHOP (JUMP) & COLLECT \\
\hline $\mathbf{L}, \mathbf{N}$ & $\mathbf{L}, \mathbf{N}$ & LIFT, POINT & LOCK,NEGATE \\
\hline Sh, Z & $\mathbf{S h}, \mathbf{Z},{ }^{\text {Voiced }} \mathbf{T h}$ & SPREAD & $\begin{array}{l}\text { PEACE \& QUIET, } \\
\text { IDENTITY }\end{array}$ \\
\hline Tz, $\mathbf{R}$ & $\mathbf{X}, \mathbf{J}, \mathbf{R}, \mathbf{Y}$ & OUT & EXCELL, \\
\hline
\end{tabular}


Semantic Keys and Reading

\begin{tabular}{|l|l|l|l||}
\hline & & & ENLIGHTEN \\
\hline $\mathbf{S}, \mathbf{G}$ & $\mathbf{S}, \mathbf{G}$ & SPIN & SEAL \\
\hline $\mathbf{D}, \mathbf{T}$ & $\mathbf{D}, \mathbf{T}, \mathbf{T h}$ & DROP & DECIDE, JUDGE \\
\hline $\mathbf{P}, \mathbf{B}, \mathbf{F}$ & $\mathbf{P}, \mathbf{B}, \mathbf{F}$ & PUSH FORWARD & BREAK \\
\hline
\end{tabular}

Individual Keys are displayed in groups. $\mathrm{K}$ is a variant of $\mathrm{C}$. Hebrew $\mathrm{CK}$-words are shown in Table 2. Their function and use is found in the process of tweaking, i.e. adjusting meaning to specific contexts.

Table 2. English/Hebrew C/K-words

\begin{tabular}{|l||l|l|l|l|l|l|l|l||}
\hline He. & Kipah & Kos & Kise & Knesiyah & Kneset & Klol & Kvosh & yaKhol \\
\hline Eng. & Skullcap & Glass & Chair & Church & Congress & InClude & Conquer & Capable \\
\hline
\end{tabular}

\subsection{Tweaking \& Super-Tweaking}

The Power but also the great challenge of qTheory, especially as a practical tool comes from tweaking. Even bright students can be frightened by the need for tweaking, which makes qTheory a "thinking person's language system".

But tweaking is something we all do in our native language, 24/7, 365 days a year. Outside of language, we also do it, as part of our Gestalt-based search for meaning. Meaning and the search for meaning is the meaning of life, the defining characteristic of homo sapiens. (Animals also think, of course, although they are severely limited in their metaphoric abilities, which are central to human language.

Tweaking is harder in a foreign language, especially when we try to be linear and mechanical, preferring memory to thinking, although we know from cognitive psychology that comprehension is a better, more effective tool for memorizing than rote memorization.

For example, how well and for how long can you memorize a long arbitrary numerical sequence like 100816449362516841 ? It is a trivially easy act of memory if you see the pattern: this is a series of square numbers. If you analyze it as such you even see the one irregularity (mistake), which also becomes easy to learn exactly because it does not fit the pattern.

Vocabulary is the classic subject of rote memorization, with flash-cards, lists, and other not-very-effective tricks. qTheory offers the ultimate "trick", the best kind of trick because it engages learners cognitively, forcing them to think out meaningful relationships, which mirror meaning change in language. These guesses are often correct, and in any case the word is learned easily when the answer is given after these guesses, qTheory to be literal, mechanical in a way it cannot be. qTheory is not a "generative" (predictive) theory, it does not predict meanings, but astute learners will sometimes, even often be able to guess the meaning of unknown words in context, far better than by context alone. This is clearly seen over many years of teaching reading comprehension in Hebrew. When students are forced 
to give one or (better) two guesses for the meaning of every single unknown word, one of their guesses is often correct, especially when two or more learners work together. In any case, they learn the word easily when it is supplied in response to such guesses, as opposed to "linear" dictionary look-up.

You gotta have SOLE! The learning becomes a SOLE: a Self-Organized Learning Experience.

\subsection{Scholars assessing qTheory}

Scholars are fairly likely to turn linear too, rather than read and learn to use a dynamic system like qTheory. This explains the frequency with articles on qTheory have been misunderstood by linguistic scholars, presenting the typical aura of reactions (neophobia) to a radically new paradigm, that may presage a new scientific revolution within linguistics, allowing linguists to study meaning directly as they have never before been able to do.

It is my hope that scholars can use obvious examples like: Down, Dumb, Die; Feisty, Fly, Force; Road, Run, aRea; etc. to propel themselves into an understanding of the system. But it is undoubtedly easier to simply resist, and dismiss the system. How did Galileo's nemesis, Pope Urban, manage not so see that the Earth revolved around the Sun? Answer: he closed his eyes (for several hundred years)!

\subsection{Graphic vs. Kinesthetic values}

While the kinesthetic value of $K$ as 'contain' is not obvious, the iconic nature of the letter is very obvious: Hebrew $\mathrm{K}$ looks like a backwards $\mathrm{C}$, giving mirror image like Kipah ("Cipah" backwards) and Cap. More typical Keys are and Sh and L. Sh seems onomatopoetic, with its Main Meaning (in fact, mm2) of PEACE \& QUIET, clearly exemplified in the Hebrew Sabbath greeting Shabat Shalom! 'Sabbath of Peace!': "Sh! it's Shabat!"

But L does not sound like its meaning Lift, but it does represent LIFTING kinesthetically, in that the tongue LIFTS to pronounce $\mathrm{L}$.

Mirror-images extend even to Hebrew 'nation' and Greek Genos 'nation', since Hebrew Gimal and Greek Gamma are mirror-images. Sh is represented in Cyrillic Sh and Russian clothing words like Shuba 'fur coat', Shapka 'cap' and others. The words do not parallel English words one by one, but the use of $S h$ for clothing words is (accidentally) characteristic of English and Russian, but not Hebrew.

The kinesthetic value of $K$ is more obvious in this Key's mml: CUT, and counterintuitive in $\mathrm{K} 2$ 'Collect'. But the second meaning of $\mathrm{K}$ is easily seen as tweaked from its first Main Meaning, CUT. After all, you can form a group only by cutting out non-members.

\section{Nonkeys}

As noted, theory was first formulated for Hebrew, naturally so, since Semantic Keys are 
exceptionally obvious in Hebrew. Nowhere is this clearer than in the notion of "Non-key" letters, first of all including vowels which aren't letters at all, but rather optional diacritics. Lest it be thought that the "consonantal preference" of qTheory is a Hebrew bias, note that speedwriting, (i.e. spdwrtng) an alternative stenographic system fur note-taking uses only consonants: spdwrtng 's $n t d f f c l t ~ t$ ' ' $n d r s t n d$, 'vn 'ftr 'Ing tm. ${ }^{\mathbb{1}}$ Speedwriting Is essentially Hebrew spelling in English. Of course it cannot work using vowels alone, in English or any language (except perhaps Hawaiian).

Other nonkeys are the "silent consonants" of Hebrew, and also other "weak" consonants ("vowel letters" y, w), "silent letters" ( ${ }^{a}$ alef, ayin, $h$ ), and also $n$. Finally $t$, (vs. "emphatic" $T$ ) occurs frequently in affixes, which are themselves nonkeys; the analysis applies primarily to roots, and affixes are initially ignored, But they also fit the general system. The most striking example of this is $\mathrm{M}$, in the Hebrew plural ending -iM (Eng. cheruniM) and 'aM 'people', Chinese Min 'people' (and the plural ending -men) and Eng. Many, Much, Russian Mnogo 'Much, Many'.

\subsection{Nonkeys as second-tier Keys}

As these last examples hint, the situation is actually more complex, to the extent that "nonkey" consonants can actually function as Keys, first, when a root has no Key, the first consonant will fill in. Thus Waw [vav] means 'hook', revealing mm2 of $\sqrt{ } \mathrm{W}$.

Secondly, after analysis of the root, affixes can be analyzed, and their meanings conform to the meanings established for (primary) Key-consonants.

Thus a atah 'you' has the Key-consonant t 'other', and means 'you' (Compare Latin Tu).

\subsection{Multi-ambiguous M}

The Hindu mantra om is often regarded as meaningless, but in qTheory it is rather muli-s,biguous, including both "MINI-MAX" $\sqrt{ } \mathrm{M}$ (SMALL \& LARGE, mediated by MEASURE): Greek micro, Macro, Metron, Russian Malo 'little/few', Mnogo, 'Much/Many', Mera 'Measure'; Hebrew $M^{o} a T$ 'little, few', Me ah 'hundred', $M^{a o} o d$ 'very', Mdod 'measure'.

But the total range of meanings of $\sqrt{M}$ is broader:

mm1: PULL (including: WATER, MASTER, KILL/DEAD); and

mm2: TOGETHER (including: WITH, MULTITUDE, MANY).

Similarly, the prefix M- means 'from' or 'causative', both from M1 'PULL' (along preposition iM means 'if'); but the suffix -iM 'plural' (as in cherubim, seraphim means 'plural'), and represents M2 'TOGETHER' as also in 'o $a M$ 'people' ${ }^{\circ}$ im 'with', cf. $M e^{a} a h$

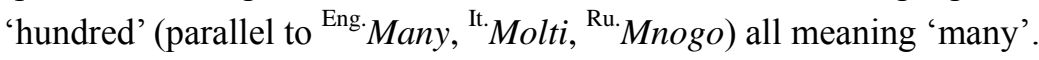

\footnotetext{
(1) Speedwriting shows the importance of "silent consonants", for which some use an apostrophe. Others don't bother with this complication, and manage effectively.
} 
The crossing between various parts speech and "particles" of speech is far more pervasive in Semitic vs. Indo-European, e.g. Waw 'hook' is used in truncated forms wa-, w'- for 'and' (cf. bar-Lev \& Palacas, 1980).

\section{The Hebrew program}

Hebrew curricula and textbooks used at two universities and in private and synagogue courses for Hebrew over several decades have used the Key-letter system. They are published by Cognella Academic Press.

\subsection{Explanation of sound change \& Equivalents}

Key consonants fall into groups (qGroups) based on similarity of sound and parallel meanings. Since the system is based in Hebrew, it is of course consistent with sound change within Hebrew, e.g. P > F, B > V, and also W > V. But some qGroups are not phonetic: $S$ and $G$.

What is more, generally Theory thus explains sound change by predicting that sound change usually follows qGroups, so that sound change usually doesn't change or disrupt meaning, So when we posit universality, the system predicts (explains) sound change, e.g. why $\mathrm{P}$ often alternates with $\mathrm{F}$ and rarely with $\mathrm{K}$ (Rumanian noapte from nocte is the rare exception).

\subsection{Key-letters are represented by capital letters}

Key-letters are represented by capital letters in examples and their translations here. This convention displays the primary argument for the universality of the posited Key-consonants in all the tables.

The claimed universality depends on equivalents across languages. Some of these equivalents are phonetically obvious, but others are not. Universality in each case depends on cross-linguistic parallels. We exemplify with obvious B vs. non-obvious $\mathrm{J} / \mathrm{X}$ and Hebrew Tz. Such sets, within a language and across languages, are here called 'octopi'.

Table 3. $\sqrt{\mathrm{B}}$ in Hebrew/English.

\begin{tabular}{|l||l|l|l|l|l||}
\hline \hline Hebrew & Barukh & Baqar & Behemah & Bneh & $\ldots$ \\
\hline English & Blessed & Beef & Beast & Build & $\ldots$ \\
\hline
\end{tabular}

Table 4. $\sqrt{ }$ Tz in Hebrew \& English $\sqrt{ } \mathrm{X} / \mathrm{J}$.

\begin{tabular}{|l||l|l|l|l|l|c|}
\hline \hline Hebrew & Tze & meTzuyan & Tzahov & Tzoair & Tzedeq & $\ldots$ \\
\hline English & eXit & eXceptional & Jaundiced & Juvenile & Justice & $\ldots$ \\
\hline
\end{tabular}

\section{Parallels, the "same-Key strategy" and the D.O.E.}

The "same-Key-consonant" strategy is an easy, useful strategy for learning words in a foreign language, which does not interfere when it doesn’t apply. 


\subsection{The D.O.E.}

The "Design of Experiment" (D.O.E.) is a statistical method used in engineering, whereby specific data points are chosen to represent different parts of data space, rather than exhaustive tracking of all data. According to this method, we would expect two different languages to have an average of 1.9 parallel words per letter. In fact, however, English and Hebrew, actually unrelated languages, exhibit 4-5 or more parallels for each letter, as exemplified, this includes not only common letters like B and R, but also less frequent letters like X, J, and Hebrew Tz. The D.O.E suggests that Parallel "octopi" of words are not coincidence, but rather the results of systematic relationships within languages, as predicted by nanosemantics-relationships that go far beyond comparative-historical linguistics.

Table 5. Some Key-letter Parallels.

\begin{tabular}{|l|l|l|l|l|c|}
\hline \multirow{2}{*}{} & Greek & Russian & Hebrew & Hebrew & $\ldots$ \\
\cline { 2 - 6 } & Biblos & Bol'shoy & Gdal & Gadol & $\ldots$ \\
\hline English & Book & Big & Grow & Great & $\ldots$ \\
\hline
\end{tabular}

\subsection{Non-parallels}

Non-parallels are actually more relevant to linguistic theory. For example, Hebrew ba $\sum a r$ 'meat' has no English parallel, but has parallels Baqar 'beef' and Behemaah 'beast' as "neighbors" in the table, i.e. in the "octopi" of parallel words. The non-parallels reveal the meanings of the Keys themselves. (But also, for example, Chinese Ping and English Peace although exceptional as variants of $\sqrt{\mathrm{P}}$ (which means PUSH FORWARD), exemplifies the "Same-key" strategy.)

\subsection{Chinese \& Japanese}

While a comprehensive analysis of Chinese and Japanese alongside the Global Alphabet would be interesting, it is sufficient for now, not to prove universality definitively but to establish a prima-facie case for it as an interesting hypothesis, to find surprising specific parallels. For example, $\mathrm{L}$ in $o L d$ is an interesting stretch of $\sqrt{\mathrm{L}}$ as LIFT, HIGH, TALL, ${ }^{(1)}$ its presence in Laod-dr (the author of Daoism) meaning 'oLd-one' seems to be a notable coincidence. But when a coincidence emerges from a coherent, articulated theory, it is not a guess, but confirmation of the theory. For example, when a ballistics expert correctly predicts where a missile will land it is not a guess but confirmation of ballistic theory. But we shall now see this kind if "coincidence" in Japanese.

One of the oddest keys is $\sqrt{ } \mathrm{N}$, meaning both FOCUS and NEGATION, two oddly different, even opposite meanings. When we find the same meanings in Japanese $\mathrm{N}$, this is (most probably) support for nanosemantics, rather than a coincidence. Neither is this surprising

\footnotetext{
(1) Size is commonly used as a metaphor for age, as in big brother or big girls don't cry.
} 
for a theory that was developed for Hebrew, but suddenly found to apply to English.

Table 6. $\sqrt{ } \mathrm{N}$ in Hebrew, Latin, \& Japanese.

\begin{tabular}{|l|c|l|l|c|}
\hline \multirow{2}{*}{} & \multicolumn{3}{|c|}{ MEANINGS } \\
\cline { 2 - 5 } & WE,US & I & NOT & IN \\
\hline Hebrew & ${ }^{a} a N u$ & ${ }^{o} a N i$ & ${ }^{a} e ́ N$ & \\
\hline Latin & Nos & & Non & $-n i$ 'at, in, on' \\
\hline Japanese & & & Ja-nai 'is-not'; -nai 'not exist' & \\
\hline
\end{tabular}

Table 7. $\sqrt{ } \mathrm{M}$ in English, Hebrew, \& Chinese

\begin{tabular}{|c|c|c|c|c|c|c|c|}
\hline & \multicolumn{7}{|c|}{ MEANINGS } \\
\hline & PULL & $\begin{array}{l}\text { MASTER, } \\
\text { MISTER }\end{array}$ & KING & $\begin{array}{l}\text { DEAD, } \\
\text { PERISH }\end{array}$ & WATER, SEA & $\begin{array}{l}\text { TOGETHER,WITH, } \\
\text { PEOPLE }\end{array}$ & PLURAL \\
\hline English & & & Monarch & Murder,Mort- & Mermaid,Marine & Mix,Marry,Match & Many,Mob \\
\hline Hebrew & M'shokh & Mar & Melekh & Met & Mayim,yaM & ${ }^{o} i M,{ }^{o} a M$ & $-i M$ \\
\hline Chinese & & & & 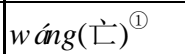 & & $\operatorname{Min}($ 民) & -men(们) \\
\hline
\end{tabular}

\section{Summary}

qTheory, nanosemantics, is a radical, even "heretical" theory of lexical semantics, positing $23^{(2)}$ Semantic Keys with kinesthetic-iconic values, which explain hundreds of relationships that have been "hiding in plain sight", in various genetically unrelated languages. To "prove" definitively their universality would require long study of dozens of Individual lexicons, so we have here just made a "prima facie" case for positing the theory. My hope is that the brevity of this article might tempt otherwise skeptical scholars to try the more significant intellectual challenge of giving it a really serious "test drive".

\section{References}

bar-Lev, Zev. \& A. Palacas. 1980. Semantic Command over Pragmatic Priority [J]. Lingua 51:467-490. bar-Lev, Zev. 2005. Arabic Key-Consonants [J]. Journal of Arabic \& Islamic Studies, 6(6):24-63.

Starke, Michal. 2009. Nanosyntax: A Short Primer to a new approach to language [OL]. URL: https://lingauf.net. [8/25/2016]

\footnotetext{
(1) The wáng(亡) is the morpheme which initial consonant is $M$ - in ancient Chinese.

(2) Table 1 presents the latest simplification, with just eight qLetters (some representing groups).
} 